\title{
Immunity against Helminths: Interactions with the Host and the Intercurrent Infections
}

\author{
Emmanuelle Moreau and Alain Chauvin \\ UMR ENVN/INRA 1300 Bioagression, Épidémiologie et Analyse de Risques, Atlanpole-La Chantrerie, \\ Route de Gachet, BP40706, 44307 Nantes, France \\ Correspondence should be addressed to Emmanuelle Moreau, moreau@vet-nantes.fr
}

Received 28 July 2009; Accepted 25 November 2009

Academic Editor: Luis I. Terrazas

Copyright (๑) 2010 E. Moreau and A. Chauvin. This is an open access article distributed under the Creative Commons Attribution License, which permits unrestricted use, distribution, and reproduction in any medium, provided the original work is properly cited.

\begin{abstract}
Helminth parasites are of considerable medical and economic importance. Studies of the immune response against helminths are of great interest in understanding interactions between the host immune system and parasites. Effector immune mechanisms against tissue-dwelling helminths and helminths localized in the lumen of organs, and their regulation, are reviewed. Helminth infections are characterized by an association of Th2-like and Treg responses. Worms are able to persist in the host and are mainly responsible for chronic infection despite a strong immune response developed by the parasitized host. Two types of protection against the parasite, namely, premune and partial immunities, have been described. Immune responses against helminths can also participate in pathogenesis. Th2/Treg-like immunomodulation allows the survival of both host and parasite by controlling immunopathologic disorders and parasite persistence. Consequences of the modified Th2-like responses on coinfection, vaccination, and inflammatory diseases are discussed.
\end{abstract}

\section{Introduction}

Many species of helminths are parasitic multicellular organisms of medical and economic importance as they infect humans and animals and sometimes provoke fatal diseases such as schistosomiasis. They can also be responsible for economic losses due to decreased milk or meat production and the cost of anthelminthic treatments of parasitized individuals. Helminths are extremely diverse. They can be classified into 3 taxonomic groups, that is, nematodes, trematodes, and cestodes, often with very different parasitic cycles. For example, (i) they may be transmitted orally (Strongyles, Fasciola sp., etc.) by transcutaneous route (Ankylostoma sp., Strongyloïdes sp., Schistosoma sp., etc.) by an arthropod vector (Onchocerca volvulus, Dirofilaria immitis, etc.); (ii) their definitive and intermediate hosts range from mammals, birds, reptiles to fish, molluscs and arthropods, and so forth; (iii) they may be localized in organ lumen or in tissues such as gut, liver (parenchyma and bile ducts), lung, lymphatic vessels, and so forth; (iv) the successive developmental stages of parasitic species may infect different tissues and cells of different organs.
Despite this great complexity, helminths usually cause asymptomatic or subclinical chronic infection, although some parasitized individuals can suffer from severe disease which may be fatal. Indeed, worms tend to be aggregated in their distribution, with a large number of hosts harboring few parasites and few heavily infected hosts $[1,2]$. This remarkable equilibrium between most hosts and parasites is the product of long-term coevolution of the two partners and particularly of the immune defence of the host and the immune evasion of the parasite. The immune responses of the hosts to helminth infection are generally characterized by a skewed Th2-like response. Helminths have developed several means of escaping these immune responses. Recently, Maizels et al. [3] called them "masters of immunomodulation". These immunomodulatory abilities enable the worm to persist in the host and can lead to interactions with inflammatory and immune mechanisms involved in other infections or to vaccines or in allergic and autoimmune diseases. The focus in this review is on pathogenic helminths of veterinary importance, especially in Ruminants medicine, and includes Fasciola spp. and gastrointestinal nematodes. 


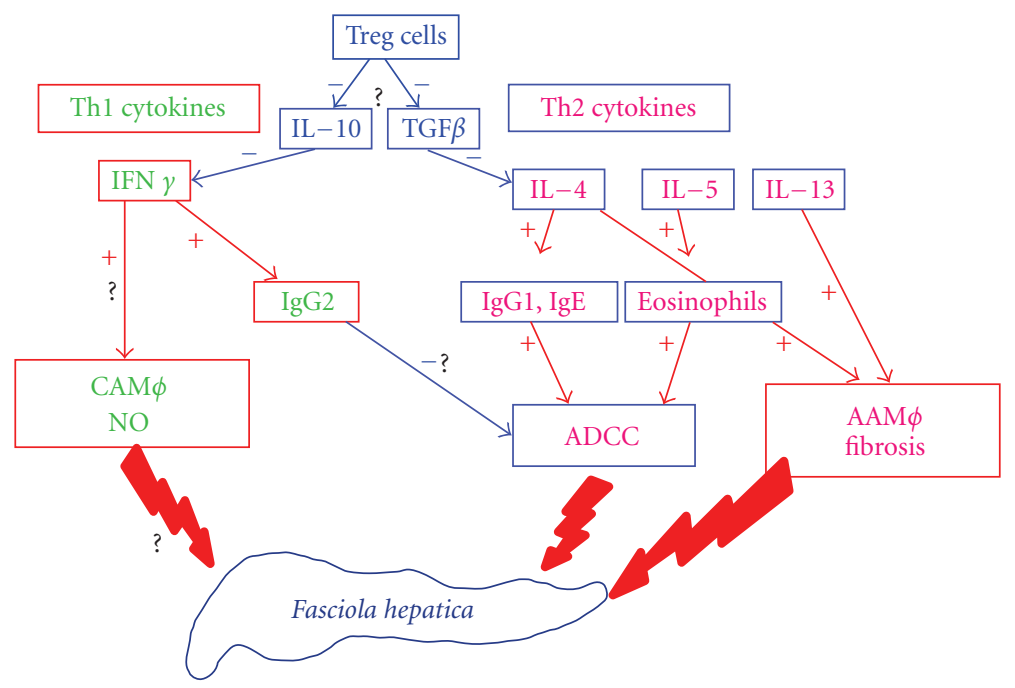

FIGURE 1: Immune mechanisms and regulation induced against Fasciola hepatica. Two main immune mechanisms are directed against $F$. hepatica in the liver parenchyma: (i) during the early phase of infection, classically activated macrophages may induce nitric oxide production which is toxic for the fluke. This mechanism needs to be confirmed and may be upregulated by Th1-type cytokines and downregulated by IL-10 produced by T regulatory cells. (ii) During the chronic phase of infection, antibody-dependent cellular cytotoxicity (ADCC) allows the release of toxic mediators such as major basic protein, eosinophil cationic protein, and reactive nitrogen intermediates. This mechanism is upregulated by Th2-type cytokines. T regulatory cells (Treg cells) produce IL-10 TGF $\beta$ which inhibit the production and function of Th1 cytokines. They downregulate any excessive Th2 response in the immunopathogenesis of fasciolosis. Finally, alternative activated macrophage $(\mathrm{AAM} \phi)$ produces molecules that are toxic to the fluke and participates in fibrosis and tissue repair.

\section{Effector Immune Mechanisms against Helminths and Their Regulation}

Helminth infections are typically associated with hypereosinophilia, considerable IgE production, mucous mastocytosis, and goblet cells hyperplasia [4]. These immune parameters are involved in different effector mechanisms highly depending on where the helminth is localized.

\subsection{Effector Mechanisms against Tissue-Dwelling Parasites and} Escape Mechanisms Developed by the Parasite. Several mechanisms against tissue-dwelling parasites have been described. These parasites are mainly larval stages, for example, of trematodes (Schistosoma spp., Fasciola spp.) or nematodes, which migrate through tissue.

Antibody Dependent Cellular Cytotoxicity (ADCC) is dependent on eosinophils, neutrophils, macrophages, or platelets as effector cells and IgE, IgG, or IgA as antibodies. The parasitic structures covered by antibodies are destroyed by cells carrying receptors to the Fc fragment $(\mathrm{RFc})$ (Figure 1). When these cells are activated by fixation of the antibodies to the RFc, they release products that are toxic to the worm (major basic protein, eosinophil cationic protein, eosinophil-derived neurotoxin, reactive nitrogen intermediates,...). ADCCs are also able to immobilize nematode larval stages as they migrate through the gut mucosa [58].

A granuloma can occur around the parasite in the tissue which stops the worm migration and development. This phenomenon has been well investigated for Schistosoma mansoni. The granuloma is composed of eosinophils, macrophages, and lymphocytes with an increasingly fibrotic extracellular matrix [4], which surrounds and segregates the eggs from the hepatic tissue [9]. In the long term, fibrosis may develop as the eggs die and the granuloma is resolved [9].

Finally, nitric oxide (NO), toxic to the worm, is released by the macrophages classically activated by IFN $\gamma$ and TNF $\alpha$ (Figure 1). This mechanism has been described mainly against trematodes (Schistosoma sp., Fasciola sp.) during acute infection, before egg production in Schistosoma mansoni $[10-12]$.

Tissue-dwelling parasites have developed several mechanisms to escape to the effector response of the host (Figure 2). For example, Fasciola sp. escapes from the immune responses by different means as follows:

(i) Fasciola gigantica produces superoxide dismutase which neutralizes superoxide radicals toxic for juveniles $[13,14]$.

(ii) F. hepatica releases cathepsin L-protease which cleaves IgE and IgG involved in the ADCC [15].

(iii) Juvenile flukes were found to be covered by IgM [16]. While eosinophils do not express $\mathrm{F} c \mu$ receptor, IgM deposition on fluke tegument could inhibit eosinophil adhesion. IgG2 produced during fasciolosis in susceptible sheep [17] has been also suspected to be a blocking immunoglobulin of the ADCC.

Furthermore, F. hepatica secretes several molecules able to modulate the immune response. Excretory-secretory products of $F$. hepatica (ESPFh) can depress the sheep and rat lymphocytes stimulation $[18,19]$ and induce eosinophil 


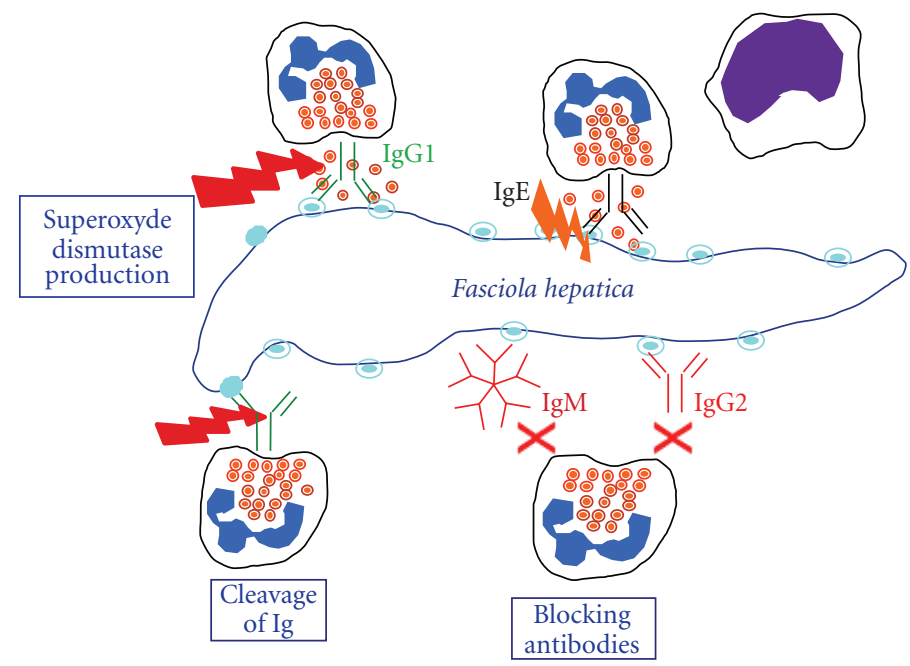

FIgURE 2: Main evasion mechanisms developed by Fasciola spp. against ADCC. Three main evasion mechanisms have been described: (i) production of superoxide dismutase and Glutathione S-transferase which neutralize superoxide radicals, (ii) cleavage of IgE and IgG involved in the ADCC, and (iii) production of blocking antibodies IgM and IgG2 which could inhibit eosinophil adhesion to flukes.

apoptosis [20]. Milbourne and Howell (1990, 1993) [21, 22] have shown that there is an "IL5-like" substance in the excretory-secretory products (ESPs) probably responsible in part of the local and systemic eosinophilia observed during fasciolosis. Cathepsin L-proteases induce a decrease of lymphoproliferation in sheep and of the CD4 expression on human and ovine $\mathrm{T}$ cells [23]. GST from F. hepatica induces a significant inhibition of nitrite production by rat peritoneal macrophages [18].

2.2. Effector Mechanisms against Parasites Localized in the Lumen of Ducts and Escape Mechanisms Developed by the Parasite. Intestinal anaphylaxis, with IgE-induced mast cells degranulation, is responsible for changes in the intestine physiology as well as architecture and chemistry of the gut epithelium, including stimulation of fluid, electrolyte and mucus secretion, smooth muscle contractility, increased vascular and epithelial permeability, and recruitment of immune cells such as eosinophil or mast cells [24] (Figure 3). This can lead to rapid elimination of the gastrointestinal larvae, before they reach their tissue niche, and to expulsion of the adult [25]. Furthermore, IgA on the surface of the gut mucosa helps to neutralize the metabolic enzymes released by digestive strongyles and interfere with the worm's ability to feed $[26,27]$.

As for tissue-dwelling parasites, parasites localized in the lumen of ducts are able to produce immunomodulatory substances to escape to the host immune responses. For example, Necator americanus secretes a metalloprotease which cleaves eotaxin, a chemotactic factor for eosinophils [28]. Gastrointestinal nematodes produce also superoxide dismutase and glutathione S-transferase which neutralize toxic oxide radicals [29]. A cystatin produced by $H$. contortus and $N$. brasiliensis modulates the antigen presentation to $\mathrm{T}$ cells by inhibiting cysteine proteases of antigen presenting cells, involved in the processing of the antigen [30, 31].

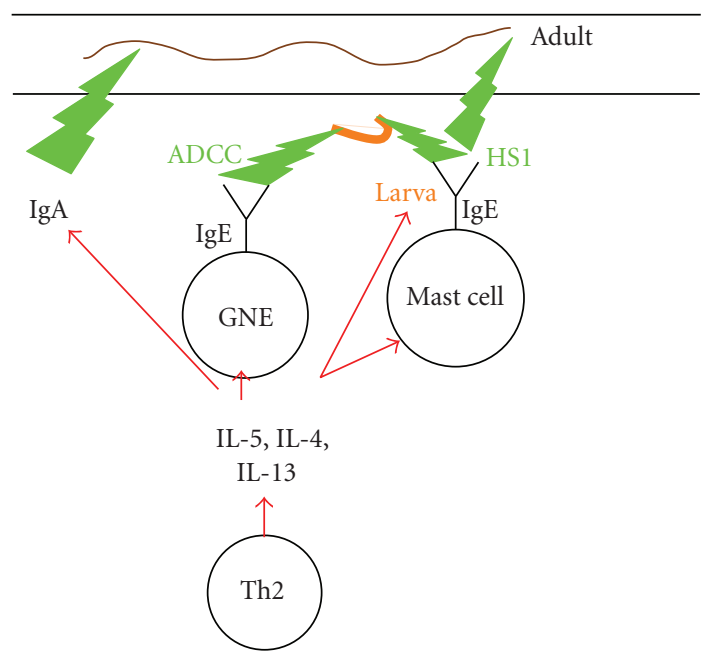

FIGURE 3: Immune response against gastrointestinal nematodes (GNE: eosinophil granulocyte, HS1: type 1 hypersensitivity). Against larva, ADCC and type 1 hypersensitivity are involved to block their migration in gut mucosa and to eliminate the parasite. Against adult in the lumen of gut, intestinal anaphylaxis responsible for muscle contractility, mucus secretion, and so forth, leads to the expulsion of the parasite. IgA neutralizes the metabolic enzymes and interferes with the worm's ability to feed.

2.3. Regulation of Immune Responses against Helminths. All these mechanisms, except the classically activated macrophages, are regulated by Th2-like cytokines and immunomodulatory cell types (Figure 1). Interleukin-4 is involved in the IgE isotype-switched B-cell responses, IL-5 is involved in the production of eosinophils, and IL-13 has similar functions to IL-4 and is involved mainly in the effector phase of inflammation and the development of fibrosis [9]. $\mathrm{T}$ regulatory cells produce the suppressive cytokines IL10 and TGF- $\beta$ which have antiinflammatory effects and 
could be involved in the skewed Th2-like responses. Immune deviation may also be promoted by the development of a Th2-driving dendritic cell population induced by excretorysecretory antigens from $N$. brasiliensis [32] or soluble egg antigen from schistosome [33]. Finally, IL-4 and IL-13 are able to alternatively activate macrophages (AAMps) which have strong antiinflammatory properties, enhance Th2 cell differentiation, contribute to fibrosis, and repair at the site of injury [34]. Thus, an environment, with downregulated proinflammatory responsiveness, activated damage repair mechanisms, and a controlled development of Th2-like antiparasite effector responses is created during infection with helminths [35].

Several proteins produced by helminths were involved in the regulation of cytokine production [36, 37]. Schistosome soluble egg antigen contains molecules as alpha-1 or omega-1 that initiate a Th2-like response [38-40]. ES62, a leucine aminopeptidase secreted by Acanthocheilonema vitae, reduces CD4+ cell IL- 4 and IFN $\gamma$ production but promotes IL-10 production by peritoneal B1 cells $[41,42]$. It also inhibits the antigen-presenting cells ability to produce IL12p70 and drives Th2-like differentiation in vitro [43, 44]. Helminths could also secrete cytokine homologues as macrophage migration inhibitory factor (MIF) which induces, with IL-4, the development of alternatively activated macrophage [45].

\section{Protective and Immunopathological Effects of the Immune Response against Helminths}

Despite the Th2-like response induced against helminths, these parasites are often able to persist in the host for a long time, resulting in chronic infection. However 2 types of immunity evaluated from the partial elimination of settled parasites and from host resistance to reinfection have been described, namely, premune immunity and partial immunity.

Premune immunity against helminths is very common and particularly observed against gastrointestinal strongyles. Premunition or concomitant immunity has been defined by MacDonald et al. [9] as a state wherein the host is protected from further infection with a given species by ongoing persistent infection with the same organism. Thus immune mechanisms existing concomitantly with parasites (adults and encysted larval stages) in animals infected by gastrointestinal nematodes prevent the establishment of new larvae. In contrast, the elimination of adult worms by the phenomenon of "self-cure" (spontaneous expulsion of adults by massive larval invasion during a short period of exposure) or by anthelminthic treatment results in the installation of new larvae until an equilibrium state is obtained. Premune immunity can also be expressed as a reduction in adult worm size and in female worm fertility. In contrast, the primary immune response against Fasciola hepatica in bovine limits the number of metacercariae which develops in adults and reduces the fertility of the females [46]. However it is unable to prevent the establishment of new parasites, which is a great difference with the premune immunity [46]. It also permits partial expulsion of adults in the bile ducts of infected cattle.
So, immune responses against Fasciola hepatica partially protect the host against the infection.

Effector mechanisms regulated by Th2-like responses play a major role in immune protection to strongyles. Comparison of the immune responses of different breeds of sheep to Haemonchus contortus revealed a greater production of Th2-like cytokines (IL-4, IL-5, IL-13) in resistant breeds [47]. Similarly, Balb/c mice, which develop a Th2-like response against Trichuris muris, were soon able to eliminate the parasite, in contrast to AKR mice which express Th1-like responses and chronic infection with the same parasite [48]. The protective role of Th1- and Th2-like responses during fluke infection is less clear: Th1-like responses might act on larvae migrating through the liver parenchyma whereas the chronic phase with F. hepatica might be due to Th2like responses against which the fluke has developed several escape mechanisms [46]. As described during infection with Schistosoma mansoni, Th2-like responses are predominant during infection by F. hepatica but early Th1-like responses seem to be involved in protection against this parasite. Indeed, sheep susceptible to F. hepatica develop a mixed Th1- and Th2-like response with IFN $\gamma$ and IL-10 production during the first 6 weeks of infection, whereas the immune response of sheep resistant to $F$. gigantica is Th1 like, with production of IFN $\gamma$ only. This suggests that protection against Fasciola $s p$. is linked with Th1-cytokine production [49]. Similarly, vaccinal trials with cathepsin L-protease from F. hepatica proved that protection induced by this antigen is mediated by a Th1-like response [50].

Although the host immune reaction against helminths may control the infection, it can also be responsible for tissue lesions and symptoms which are often the primary cause of disease during worm infection. Immunopathologic phenomena have been thoroughly investigated in infections with Schistosoma spp. As described above for F. hepatica infection, acute schistosomosis is associated with Th1-like responses against adult parasites. The Th2-like responses, induced as a result of egg antigens secretion, downregulate the production and effector functions of Th1-like mediators $[51,52]$. When Th2-like responses against the eggs were blocked experimentally, an exacerbated granuloma driven by Th1 and Th17 cells resulted in hepatic damage and death [53]. Granulomatous responses evolve from an early Th1- to a sustained and dominant Th2-like response [54]. Whereas tissue fibrosis stimulated by Th2-like cytokine (IL13) promotes tissue healing, excessive fibrosis may become pathogenic with loss of hepatic functions and portal hypertension $[54,55]$. It seems that during trematode infections Th1-like responses are more protective than Th2-like responses against which these parasites have developed many escape mechanisms. Although Th1-like responses are closely associated with immunopathogenesis, Th2-like responses may also contribute to inflammatory damage. Treg cells seem to regulate this detrimental immune response by suppressing the Th1-like response and by downregulating any excessive Th2-like response during granuloma formation [56].

Granuloma formation dependent on the Th2-like response is also observed during gastrointestinal nematode infection. A recent study has shown that experimental 
downregulation of the Th2-like response to Nippostrongylus brasiliensis suppresses resistance to gastrointestinal nematode infection, pulmonary granulomatous inflammation, and fibrosis [57]. Similarly bovine ostertagiosis is associated with diarrhoea, inappetence, and weight loss. These clinical signs could result from smooth muscle contractility, increased mucus production, loss of specialized cells in the abomasal epitheliums induced by IgE-mediated immediate hypersensitivity reactions, and degranulation of mast and goblet cells and of proinflammatory mediators [24, 58].

Hence, protection against gastrointestinal nematodes and against tissue-dwelling trematodes is controlled by Th2and Th1-like responses, respectively [59]. The migration step in tissue is considered an immunoevasive strategy due to the predominant Th2-like response during helminth infection whereas protection in tissue is mediated by the Th1-like response [59]. However, the immune mechanisms, particularly those regulated by Th1-like cytokines, are responsible for considerable immunopathological damage and for the clinical signs observed during a helminthic disease. Even if the immune responses against most of helminths are orchestrated by Th2-like cytokines, the worms are still able to persist in the host for a long time. Indeed, the immune response during the chronic phase of infection was recently reported to be a modified Th2-like response, that is, a Th2-like response associated with Treg activity and the production of antiinflammatory cytokines such as IL-10 and TGF $\beta$ [60]. The induction of immunomodulatory Th2/Treg responses would allow the survival of both partners, by downregulating the host's inflammatory response and the immunopathological lesions observed during helminth infection, and also the protective immune mechanisms directed against the parasite $[61,62]$.

\section{Helminths and Coinfection}

Some helminths are able to downregulate the Th1-like response because their high immunomodulator activity allows the induction of Th2/Treg-type responses. Indeed F. hepatica inhibits the Th1-like response induced by Bordetella pertussis in mice [63]. Similarly F. hepatica is also able to change the predictive value of the tuberculosis diagnosis test by modifying the immune response against Mycobacterium bovis [64]. Helminths can affect the evolution of coinfection by making animals more resistant to pathogens in which protection is mediated by the Th2-like response and more susceptible to pathogens in which protection is mediated by the Th1-like response [9]. For example, the expulsion of T. muris by mice is dependent on the Th2-like response. Curry et al. [65] demonstrated that mice susceptible to T. muris and coinfected with S. mansoni which induced the Th2-like response were able to eliminate T. muris by producing Th2-like cytokines. In contrast, mice infected with S. mansoni were more susceptible to Toxoplama gondii (protected by the Th1-like response) by inducing high mortality and weak production of IFN $\gamma$ and NO, compared to mice solely infected with T. gondii [66]. Helminths were also able to inhibit the development of protective immunity regulated by Th1-like cytokines against Plasmodium sp. [62].
Many analyses of helminth - Plasmodium coinfections have provided controversial results as the parasite burden of Plasmodium sp. is dependent on the helminth species used, the intensity and duration of worm infection, and the age of the individual under study [67].

Helminths influence not only host resistance to another pathogen but also the gravity of the resulting disease. Cerebral malaria is associated with an overproduction of proinflammatory cytokines. Helminth infections are able to decrease the production of these cytokines by secreting IL10 and TGF $\beta$ and thereby diminish the risk of severe disease [62]. The nematode Heligmosomoides polygyrus promotes an immune response regulated by Th2-like cytokines, alternative activated macrophages, and regulatory $\mathrm{T}$ cells, and hence, prevents the inflammatory reaction controlled by Th1 cytokines and severe immunopathological lesions observed during schistosomosis [68]. Trichinella spiralis infection limits pulmonary damage induced by influenza virus in mice [69]. In contrast, $H$. polygyrus promotes intestinal lesions due to bacteria such as Citrobacter rodentium by alternative activation of macrophages [70].

However, other pathogens can also influence the immune response against helminths. For example, Miller et al. [71] recently showed that the production of Th1-like cytokines and classic activation of macrophages were little altered when $F$. hepatica infection preceded or succeeded T. gondii infections, whereas the production of $F$. hepatica-specific Th2like cytokines and recruitment of AAMp were suppressed by $T$. gondii infection. Similarly, neutrophil-activating protein from Helicobacter pylori downmodulated the Th2-like response to Trichinella spiralis infection [72].

The effects of helminths on infections with other pathogens are complex and dependent on many factors such as the helminth species, coinfecting pathogen, protective and pathological immune mechanisms, and also the host and the individual.

\section{Helminths and Vaccination}

Several studies have shown that helminths can influence vaccine efficacy by modulating host immune response, in particular when Th1-like and cellular-dependent responses are required. Schistosoma sp. and Onchocerca volvulus infections decrease the efficacy of vaccine against tuberculosis or tetanus [62], and Ascaris suum alters the efficacy of vaccine against Mycoplasma hyopneumoniae [73]. In mice, H. polygyrus was able to downregulate the strong immunity against Plasmodium chabaudi induced by blood stage antigens [74]. Effects of helminth infections on vaccine efficacy must be taken into account when using vaccines and also when developing new vaccines, in particular by choosing adapted adjuvants which are able to counterbalance the immunomodulatory activities of the helminth.

\section{Helminths and Allergic and Autoimmune Diseases}

For several years, epidemiologic observations have shown that the prevalence of helminth infection is decreasing in 
westernized countries whereas the prevalence of diseases due to immune or inflammatory disorders such as allergic or autoimmune diseases is increasing. Epidemiologic and experimental data prove that chronic infection with helminths is protective against allergy. Humans infected with worms rarely develop allergic reactions [9] and an allergic reaction against ovalbumin was inhibited in mice infected with $H$. polygyrus or Schistosoma sp. [62, 75]. Similarly, immunization with Toxascaris leonina-derived proteins was able to inhibit allergy-specific Th2-like responses to ovalbumin [76]. Treatment against gastrointestinal nematodes increases cutaneous reactivity against house-dust mites [77]. These results are paradoxical because allergy is linked to mastocyte degranulation by IgE; the production of which is stimulated by helminths. In fact, worms induce the production of large quantities of antiinflammatory cytokines (IL-10, TGF $\beta$ ) by the regulatory $\mathrm{T}$ cells which then inhibit allergic inflammation.

In the same way, helminths can protect the host against autoimmune disease or at least decrease the gravity of symptoms induced by autoimmune inflammation. For example, S. mansoni infection inhibits the development of type 1 diabetes in NOD mice [78] or of experimental autoimmune encephalomyelitis in mice [79]. Helminth-specific Treg cells and their antiinflammatory cytokines (IL-10, TGF $\beta$ ) seem to be largely implicated in the inflammatory disorders associated with allergic diseases. Several studies are currently underway to investigate the possibility of treating allergic inflammatory diseases with immunomodulatory molecules from helminths, with special focus on the molecules involved and the ways in which helminths manipulate the host response, particularly how they activate and induce the expansion of Treg cells.

\section{Immunomodulatory Molecules of Helminths As New Antiinflammatory Drugs}

Immunomodulatory function of helminths and their products could be used as antiinflammatory drugs. Trichuris suis has been tested recently to treat patients with inflammatory bowel disease and Crohn's disease with success $[80,81]$. An excretory-secretory protein of Acanthocheilonema vitae, ES62, has been well studied for its antiinflammatory property. ES62 significantly decreases the severity of collageninduced arthritis in mice [80] and of cutaneous hypersensitivity dependant on mast cells [81]. However, these immunomodulatory molecules could have side effects by increasing the risk of infections. Furthermore, they could be responsible for allergic reactions because they could be allergens or they could cross-react with allergens derived from pollen or another source [82].

In conclusion, helminth species have coevolved with their host for a long time. This has led to a strict adaptation which enables them to settle and persist in the host. Helminths are strong immunomodulators able to interfere with immune and inflammatory mechanisms induced by themselves and by coinfecting pathogens, inflammatory disorders, or vaccine. Immunomodulatory products from helminths are probably the antiinflammatory molecules of the future. Effects of helminths on the host immune system are not properly known because data are partial or can not be generalized between species. Nevertheless these effects need to be taken in account when controlling helminths and the diseases induced by worms. However, an individual could be infected by several pathogens and it will be necessary to evaluate the overall immune equilibrium resulting from the immune interaction between host and pathogens in different tissues and organs, which is still difficult, for instance.

\section{References}

[1] S. Gaba, V. Ginot, and J. Cabaret, "Modelling macroparasite aggregation using a nematode-sheep system: the Weibull distribution as an alternative to the negative binomial distribution?" Parasitology, vol. 131, no. 3, pp. 393-401, 2005.

[2] A. Hall, S. Horton, and N. de Silva, "The costs and cost-effectiveness of mass treatment for intestinal nematode worm infections using different treatment thresholds," PLoS Neglected Tropical Diseases, vol. 3, no. 3, article e402, 2009.

[3] R. M. Maizels, A. Balic, N. Gomez-Escobar, M. Nair, M. D. Taylor, and J. E. Allen, "Helminth parasites-masters of regulation," Immunological Reviews, vol. 201, pp. 89-116, 2004.

[4] R. M. Anthony, L. I. Rutitzky, J. F. Urban Jr., M. J. Stadecker, and W. C. Gause, "Protective immune mechanisms in helminth infection," Nature Reviews Immunology, vol. 7, no. 12, pp. 975-987, 2007.

[5] M. Capron and A. Capron, "Effector functions of eosinophils in schistosomiasis," Memorias do Instituto Oswaldo Cruz, vol. 87, no. 4, pp. 167-170, 1992.

[6] E. N. T. Meeusen, A. Balic, and V. Bowles, "Cells, cytokines and other molecules associated with rejection of gastrointestinal nematode parasites," Veterinary Immunology and Immunopathology, vol. 108, no. 1-2, pp. 121-125, 2005.

[7] D. Piedrafita, J. C. Parsons, R. M. Sandeman, et al., "Antibodydependent cell-mediated cytotoxicity to newly excysted juvenile Fasciola hepatica in vitro is mediated by reactive nitrogen intermediates," Parasite Immunology, vol. 23, no. 9, pp. 473482, 2001.

[8] P. F. Weller, "Eosinophils: structure and functions," Current Opinion in Immunology, vol. 6, no. 1, pp. 85-90, 1994.

[9] A. S. MacDonald, M. I. Araujo, and E. J. Pearce, "Immunology of parasitic helminth infections," Infection and Immunity, vol. 70, no. 2, pp. 427-433, 2002.

[10] L. Cervi, G. Rossi, H. Cejas, and D. T. Masih, "Fasciola hepatica-induced immune suppression of spleen mononuclear cell proliferation: role of nitric oxide," Clinical Immunology and Immunopathology, vol. 87, no. 2, pp. 145-154, 1998.

[11] R. T. Gazzinelli, I. P. Oswald, S. L. James, and A. Sher, "IL10 inhibits parasite killing and nitrogen oxide production by IFN- $\gamma$ - activated macrophages," Journal of Immunology, vol. 148, no. 6, pp. 1792-1796, 1992.

[12] P. Sibille, O. Tliba, and C. Boulard, "Early and transient cytotoxic response of peritoneal cells from Fasciola hepaticainfected rats," Veterinary Research, vol. 35, no. 5, pp. 573-584, 2004.

[13] G. Ganga, J. P. Varshney, and R. C. Patra, "Activity of antioxidant enzymes in excretory-secretory fluid and somatic extracts of Fasciola gigantica," Journal of Veterinary Parasitology, vol. 21, no. 1, pp. 51-52, 2007. 
[14] D. Piedrafita, E. Estuningsih, J. Pleasance, et al., "Peritoneal lavage cells of Indonesian thin-tail sheep mediate antibodydependent superoxide radical cytotoxicity in vitro against newly excysted juvenile Fasciola gigantica but not juvenile Fasciola hepatica," Infection and Immunity, vol. 75, no. 4, pp. 1954-1963, 2007.

[15] A. M. Smith, A. J. Dowd, M. Heffernan, C. D. Robertson, and J. P. Dalton, "Fasciola hepatica: a secreted cathepsin Llike proteinase cleaves host immunoglobulin," International Journal for Parasitology, vol. 23, no. 8, pp. 977-983, 1993.

[16] A. Chauvin and C. Boulard, "Local immune response to experimental Fasciola hepatica infection in sheep," Parasite, vol. 3, no. 3, pp. 209-215, 1996.

[17] D. S. Hansen, D. G. Clery, S. E. Estuningsih, S. Widjajanti, S. Partoutomo, and T. W. Spithill, "Immune responses in Indonesian thin tail and Merino sheep during a primary infection with Fasciola gigantica: lack of a specific $\mathrm{IgG}_{2}$ antibody response is associated with increased resistance to infection in Indonesian sheep," International Journal for Parasitology, vol. 29, no. 7, pp. 1027-1035, 1999.

[18] L. Cervi, G. Rossi, and D. T. Masih, "Potential role for excretory-secretory forms of glutathione-S-transferase (GST) in Fasciola hepatica," Parasitology, vol. 119, no. 6, pp. 627-633, 1999.

[19] E. Moreau, S. Hervé, Z. W. Yu, and C. Alain, "Modulation of sheep lymphocyte responses by Fasciola hepatica excretorysecretory products," Veterinary Parasitology, vol. 108, no. 3, pp. 207-215, 2002.

[20] M. C. Serradell, L. Guasconi, L. Cervi, L. S. Chiapello, and D. T. Masih, "Excretory-secretory products from Fasciola hepatica induce eosinophil apoptosis by a caspase-dependent mechanism," Veterinary Immunology and Immunopathology, vol. 117, no. 3-4, pp. 197-208, 2007.

[21] E. A. Milbourne and M. J. Howell, "Eosinophil responses to Fasciola hepatica in rodents," International Journal for Parasitology, vol. 20, no. 5, pp. 705-708, 1990.

[22] E. A. Milbourne and M. J. Howell, "Eosinophil differentiation in response to Fasciola hepatica and its excretory/secretory antigens," International Journal for Parasitology, vol. 23, no. 8, pp. 1005-1009, 1993.

[23] R. K. Prowse, P. Chaplin, H. C. Robinson, and T. W. Spithill, "Fasciola hepatica cathepsin L suppresses sheep lymphocyte proliferationin vitro and modulates surface CD4 expression on human and ovine T cells," Parasite Immunology, vol. 24, no. 2, pp. 57-66, 2002.

[24] M. J. G. Farthing, "Immune response-mediated pathology in human intestinal parasitic infection," Parasite Immunology, vol. 25, no. 5, pp. 247-257, 2003.

[25] A. Balic, V. M. Bowles, and E. N. T. Meeusen, "Mechanisms of immunity to Haemonchus contortus infection in sheep," Parasite Immunology, vol. 24, no. 1, pp. 39-46, 2002.

[26] H. S. Gill, G. D. Gray, D. L. Watson, and A. J. Husband, "Isotype-specific antibody responses to Haemonchus contortus in genetically resistant sheep," Parasite Immunology, vol. 15, no. 2, pp. 61-67, 1993.

[27] G. Smith, "The population biology of the parasitic stages of Haemonchus contortus," Parasitology, vol. 96, no. 1, pp. 185195,1988

[28] F. J. Culley, A. Brown, D. M. Conroy, I. Sabroe, D. I. Pritchard, and T. J. Williams, "Eotaxin is specifically cleaved by hookworm metalloproteases preventing its action in vitro and in vivo," Journal of Immunology, vol. 165, no. 11, pp. 64476453, 2000 .
[29] S. Liddell and D. P. Knox, "Extracellular and cytoplasmic $\mathrm{Cu} / \mathrm{Zn}$ superoxide dismutases from Haemonchus contortus," Parasitology, vol. 116, no. 4, pp. 383-394, 1998.

[30] G. F. J. Newlands, P. J. Skuce, D. P. Knox, and W. D. Smith, "Cloning and expression of cystatin, a potent cysteine protease inhibitor from the gut of Haemonchus contortus," Parasitology, vol. 122, no. 3, pp. 371-378, 2001.

[31] T. Dainichi, Y. Maekawa, K. Ishii, et al., "Nippocystatin, a cysteine protease inhibitor from Nippostrongylus brasiliensis, inhibits antigen processing and modulates antigen-specific immune response," Infection and Immunity, vol. 69, no. 12, pp. 7380-7386, 2001.

[32] K. J. Else, "Have gastrointestinal nematodes outwitted the immune system?" Parasite Immunology, vol. 27, no. 10-11, pp. 407-415, 2005.

[33] P. G. Thomas, M. R. Carter, O. Atochina, et al., "Maturation of dendritic cell 2 phenotype by a helminth glycan uses a Toll-like receptor 4-dependent mechanism," Journal of Immunology, vol. 171, no. 11, pp. 5837-5841, 2003.

[34] T. Kreider, R. M. Anthony, J. F. Urban Jr., and W. C. Gause, "Alternatively activated macrophages in helminth infections," Current Opinion in Immunology, vol. 19, no. 4, pp. 448-453, 2007.

[35] J. A. Jackson, I. M. Friberg, S. Little, and J. E. Bradley, "Review series on helminths, immune modulation and the hygiene hypothesis: immunity against helminths and immunological phenomena in modern human populations: coevolutionary legacies?" Immunology, vol. 126, no. 1, pp. 18-27, 2009.

[36] D. A. Harn, J. McDonald, O. Atochina, and A. A. Da'dara, "Modulation of host immune responses by helminth glycans," Immunological Reviews, vol. 230, no. 1, pp. 247-257, 2009.

[37] J. P. Hewitson, J. R. Grainger, and R. M. Maizels, "Helminth immunoregulation: the role of parasite secreted proteins in modulating host immunity," Molecular and Biochemical Parasitology, vol. 167, no. 1, pp. 1-11, 2009.

[38] J.-M. Grzych, E. Pearce, A. Cheever, et al., "Egg deposition is the major stimulus for the production of Th2 cytokines in murine Schistomiasis mansoni," Journal of Immunology, vol. 146, no. 4, pp. 1322-1327, 1991.

[39] G. Schramm, F. H. Falcone, A. Gronow, et al., "Molecular characterization of an interleukin-4-inducing factor from Schistosoma mansoni eggs," Journal of Biological Chemistry, vol. 278, no. 20, pp. 18384-18392, 2003.

[40] B. Everts, G. Perona-Wright, H. H. Smits, et al., "Omega-1, a glycoprotein secreted by Schistosoma mansoni eggs, drives Th2 responses," Journal of Experimental Medicine, vol. 206, no. 8, pp. 1673-1680, 2009.

[41] E. H. Wilson, E. Katz, H. S. Goodridge, M. M. Harnett, and W. Harnett, "In vivo activation of murine peritoneal B1 cells by the filarial nematode phosphorylcholine-containing glycoprotein ES-62," Parasite Immunology, vol. 25, no. 8-9, pp. 463-466, 2003.

[42] F. A. Marshall, A. M. Grierson, P. Garside, W. Harnett, and M. M. Harnett, "ES-62, an immunomodulator secreted by filarial nematodes, suppresses clonal expansion and modifies effector function of heterologous antigen-specific $\mathrm{T}$ cells in vivo," Journal of Immunology, vol. 175, no. 9, pp. 5817-5826, 2005.

[43] H. S. Goodridge, W. Harnett, F. Y. Liew, and M. M. Harnett, "Differential regulation of interleukin-12 p40 and p35 induction via Erk mitogen-activated protein kinase-dependent and -independent mechanisms and the implications for bioactive IL-12 and IL-23 responses," Immunology, vol. 109, no. 3, pp. 415-425, 2003. 
[44] M. Whelan, M. M. Harnett, K. M. Houston, V. Patel, W. Harnett, and K. P. Rigley, "A filarial nematode-secreted product signals dendritic cells to acquire a phenotype that drives development of Th2 cells," Journal of Immunology, vol. 164, no. 12, pp. 6453-6460, 2000.

[45] L. Prieto-Lafuente, W. F. Gregory, J. E. Allen, and R. M. Maizels, "MIF homologues from a filarial nematode parasite synergize with IL-4 to induce alternative activation of host macrophages," Journal of Leukocyte Biology, vol. 85, no. 5, pp. 844-854, 2009.

[46] A. Chauvin, W. Zhang, and E. Moreau, "Fasciolosis of ruminants: immunity, immunomodulation and control strategies," Bulletin de l'Academie Veterinaire de France, vol. 160, pp. 8592, 2007.

[47] G. Terefe, C. Lacroux, O. Andreoletti, et al., "Immune response to Haemonchus contortus infection in susceptible (INRA 401) and resistant (Barbados Black Belly) breeds of lambs," Parasite Immunology, vol. 29, no. 8, pp. 415-424, 2007.

[48] W. C. Gause, J. F. Urban Jr., and M. J. Stadecker, "The immune response to parasitic helminths: insights from murine models," Trends in Immunology, vol. 24, no. 5, pp. 269-277, 2003.

[49] W. Y. Zhang, E. Moreau, J. C. Hope, C. J. Howard, W. Y. Huang, and A. Chauvin, "Fasciola hepatica and Fasciola gigantica: comparison of cellular response to experimental infection in sheep," Experimental Parasitology, vol. 111, no. 3, pp. 154-159, 2005.

[50] G. Mulcahy and J. P. Dalton, "Cathepsin L proteinases as vaccines against infection with Fasciola hepatica (liver fluke) in ruminants," Research in Veterinary Science, vol. 70, no. 1, pp. 83-86, 2001.

[51] M. H. Kaplan, J. R. Whitfield, D. L. Boros, and M. J. Grusby, "Th2 cells are required for the Schistosoma mansoni egginduced granulomatous response," Journal of Immunology, vol. 160, no. 4, pp. 1850-1856, 1998.

[52] E. J. Pearce, C. M. Kane, J. Sun, J. J. Taylor, A. S. McKee, and L. Cervi, "Th2 response polarization during infection with die helminth parasite Schistosoma mansoni," Immunological Reviews, vol. 201, pp. 117-126, 2004.

[53] M. J. Stadecker, H. Asahi, E. Finger, H. J. Hernandez, L. I. Rutitzky, and J. Sun, "The immunobiology of Th1 polarization in high-pathology schistosomiasis," Immunological Reviews, vol. 201, pp. 168-179, 2004.

[54] T. A. Wynn, R. W. Thompson, A. W. Cheever, and M. M. Mentink-Kane, "Immunopathogenesis of schistosomiasis," Immunological Reviews, vol. 201, pp. 156-167, 2004.

[55] E. J. Pearce and A. S. MacDonald, "The immunobiology of schistosomiasis," Nature Reviews Immunology, vol. 2, no. 7, pp. 499-511, 2002.

[56] M. L. Burke, M. K. Jones, G. N. Gobert, Y. S. Li, M. K. Ellis, and D. P. McManus, "Immunopathogenesis of human schistosomiasis," Parasite Immunology, vol. 31, no. 4, pp. 163176, 2009.

[57] J. T. Pesce, T. R. Ramalingam, M. S. Wilson, et al., "Retnla (Relm $\alpha /$ Fizz1) suppresses helminth-induced Th2type immunity," PLoS Pathogens, vol. 5, no. 4, Article ID e1000393, 2009.

[58] G. Mulcahy, S. O’Neill, S. Donnelly, and J. P. Dalton, "Helminths at mucosal barriers-interaction with the immune system," Advanced Drug Delivery Reviews, vol. 56, no. 6, pp. 853-868, 2004.

[59] G. Mulcahy, S. O’Neill, J. Fanning, E. McCarthy, and M. Sekiya, "Tissue migration by parasitic helminths — an immunoevasive strategy?” Trends in Parasitology, vol. 21, no. 6, pp. 273-277, 2005.
[60] R. M. Maizels, "Infections and allergy-helminths, hygiene and host immune regulation," Current Opinion in Immunology, vol. 17, no. 6, pp. 656-661, 2005.

[61] R. M. Maizels and M. Yazdanbakhsh, "Immune regulation by helminth parasites: cellular and molecular mechanisms," Nature Reviews Immunology, vol. 3, no. 9, pp. 733-744, 2003.

[62] E. Van Riet, F. C. Hartgers, and M. Yazdanbakhsh, "Chronic helminth infections induce immunomodulation: consequences and mechanisms," Immunobiology, vol. 212, no. 6, pp. 475-490, 2007.

[63] S. M. O’Neill, K. H. G. Mills, and J. P. Dalton, “Fasciola hepatica cathepsin L cysteine proteinase suppresses Bordetella pertussis-specific interferon- $\gamma$ production in vivo," Parasite Immunology, vol. 23, no. 10, pp. 541-547, 2001.

[64] R. J. Flynn, C. Mannion, O. Golden, O. Hacariz, and G. Mulcahy, "Experimental Fasciola hepatica infection alters responses to tests used for diagnosis of bovine tuberculosis," Infection and Immunity, vol. 75, no. 3, pp. 1373-1381, 2007.

[65] A. J. Curry, K. J. Else, F. Jones, A. Bancroft, R. K. Grencis, and D. W. Dunne, "Evidence that cytokine-mediated immune interactions induced by Schistosoma mansoni alter disease outcome in mice concurrently infected with Trichuris muris," Journal of Experimental Medicine, vol. 181, no. 2, pp. 769-774, 1995.

[66] A. J. Marshall, L. R. Brunet, Y. van Gessel, et al., "Toxoplasma gondii and Schistosoma mansoni synergize to promote hepatocyte dysfunction associated with high levels of plasma TNF- $\alpha$ and early death in C57BL/6 mice," Journal of Immunology, vol. 163, no. 4, pp. 2089-2097, 1999.

[67] S. Specht and A. Hoerauf, "Does helminth elimination promote or prevent malaria?" The Lancet, vol. 369, no. 9560, pp. 446-447, 2007.

[68] L. E. Bazzone, P. M. Smith, L. I. Rutitzky, et al., "Coinfection with the intestinal nematode Heligmosomoides polygyrus markedly reduces hepatic egg-induced immunopathology and proinflammatory cytokines in mouse models of severe schistosomiasis," Infection and Immunity, vol. 76, no. 11, pp. 5164-5172, 2008.

[69] R. C. Furze, T. Hussell, and M. E. Selkirk, "Amelioration of influenza-induced pathology in mice by coinfection with Trichinella spiralis," Infection and Immunity, vol. 74, no. 3, pp. 1924-1932, 2006.

[70] M. Weng, D. Huntley, I.-F. Huang, et al., "Alternatively activated macrophages in intestinal helminth infection: effects on concurrent bacterial colitis," Journal of Immunology, vol. 179, no. 7, pp. 4721-4731, 2007.

[71] C. M. D. Miller, N. C. Smith, R. J. Ikin, N. R. Boulter, J. P. Dalton, and S. Donnelly, "Immunological interactions between 2 common pathogens, Th1-inducing protozoan Toxoplasma gondii and the Th2-inducing helminth Fasciola hepatica," PLoS ONE, vol. 4, no. 5, article e5692, 2009.

[72] G. Del Prete, L. Chiumiento, A. Amedei, et al., "Immunosuppression of $\mathrm{TH} 2$ responses in Trichinella spiralis infection by Helicobacter pylori neutrophil-activating protein," Journal of Allergy and Clinical Immunology, vol. 122, no. 5, pp. 908-913, 2008.

[73] J. F. Urban Jr., N. R. Steenhard, G. I. Solano-Aguilar, et al., "Infection with parasitic nematodes confounds vaccination efficacy," Veterinary Parasitology, vol. 148, no. 1, pp. 14-20, 2007.

[74] Z. Su, M. Segura, and M. M. Stevenson, "Reduced protective efficacy of a blood-stage malaria vaccine by concurrent nematode infection," Infection and Immunity, vol. 74, no. 4, pp. 2138-2144, 2006. 
[75] M. S. Wilson, M. D. Taylor, A. Balic, C. A. M. Finney, J. R. Lamb, and R. M. Maizels, "Suppression of allergic airway inflammation by helminth-induced regulatory T cells," Journal of Experimental Medicine, vol. 202, no. 9, pp. 11991212, 2005.

[76] K. H. Lee, H. K. Park, H. J. Jeong, et al., "Immunization of proteins from Toxascaris leonina adult worm inhibits allergic specific Th2 response," Veterinary Parasitology, vol. 156, no. 34, pp. 216-225, 2008.

[77] A. H. J. van den Biggelaar, L. C. Rodrigues, R. Van Ree, et al., "Long-term treatment of intestinal helminths increases mite skin-test reactivity in Gabonese schoolchildren," Journal of Infectious Diseases, vol. 189, no. 5, pp. 892-900, 2004.

[78] A. Cooke, P. Tonks, F. M. Jones, et al., "Infection with Schistosoma mansoni prevents insulin dependent diabetes mellitus in non-obese diabetic mice," Parasite Immunology, vol. 21, no. 4, pp. 169-176, 1999.

[79] D. Sewell, Z. Qing, E. Reinke, et al., "Immunomodulation of experimental autoimmune encephalomyelitis by helminth ova immunization," International Immunology, vol. 15, no. 1, pp. 59-69, 2003.

[80] R. W. Summers, D. E. Elliott, K. Qadir, J. F. Urban Jr., R. Thompson, and J. V. Weinstock, "Trichuris suis seems to be safe and possibly effective in the treatment of inflammatory bowel disease," American Journal of Gastroenterology, vol. 98, no. 9, pp. 2034-2041, 2003.

[81] R. W. Summers, D. E. Elliot, J. F. Urban Jr., R. Thompson, and J. V. Weinstock, "Trichuris suis therapy in Crohn's disease," Gut, vol. 54, no. 1, pp. 87-90, 2005.

[82] W. Harnett, I. B. McInnes, and M. M. Harnett, "ES-62, a filarial nematode-derived immunomodulator with antiinflammatory potential," Immunology Letters, vol. 94, no. 1-2, pp. 27-33, 2004. 

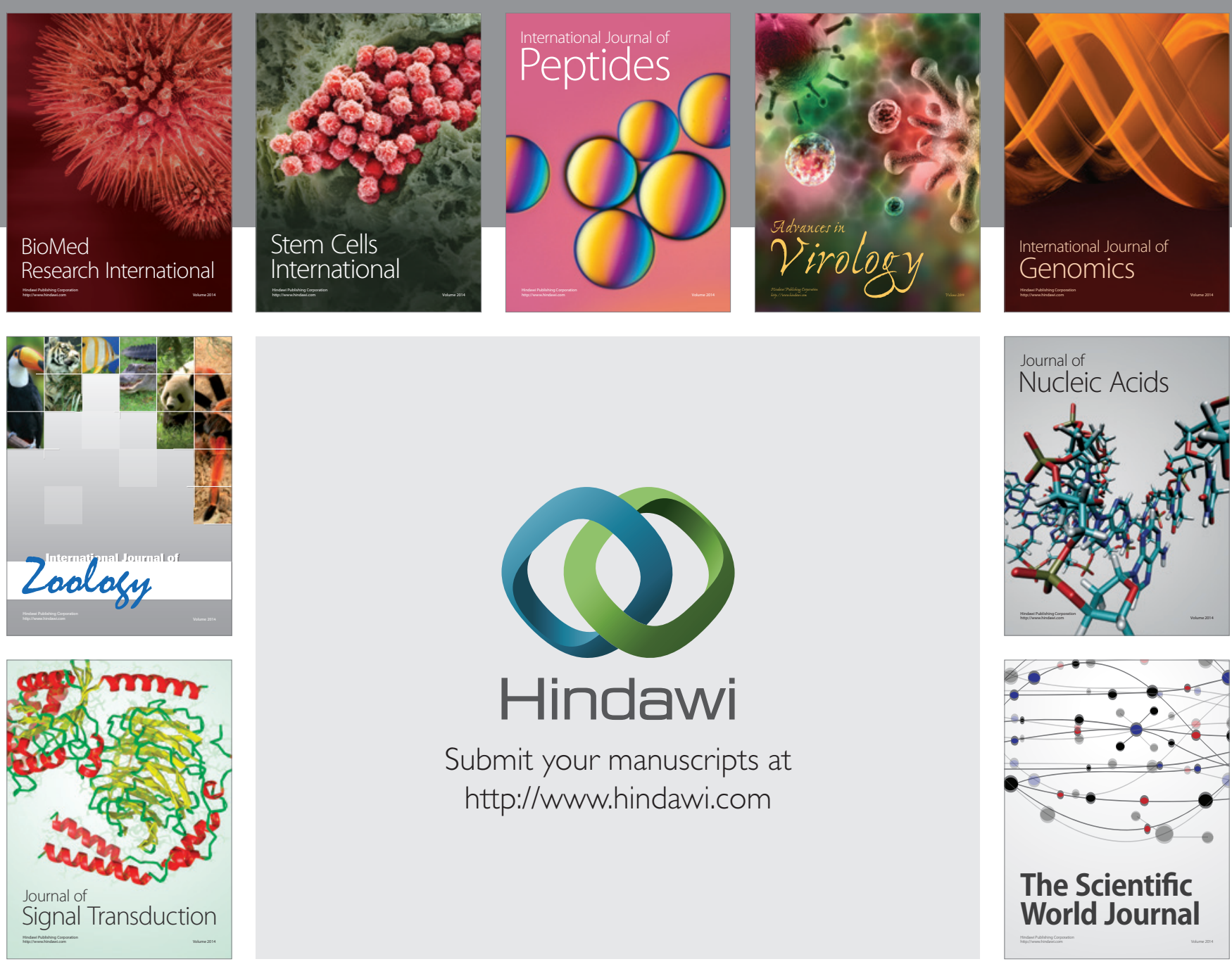

Submit your manuscripts at

http://www.hindawi.com
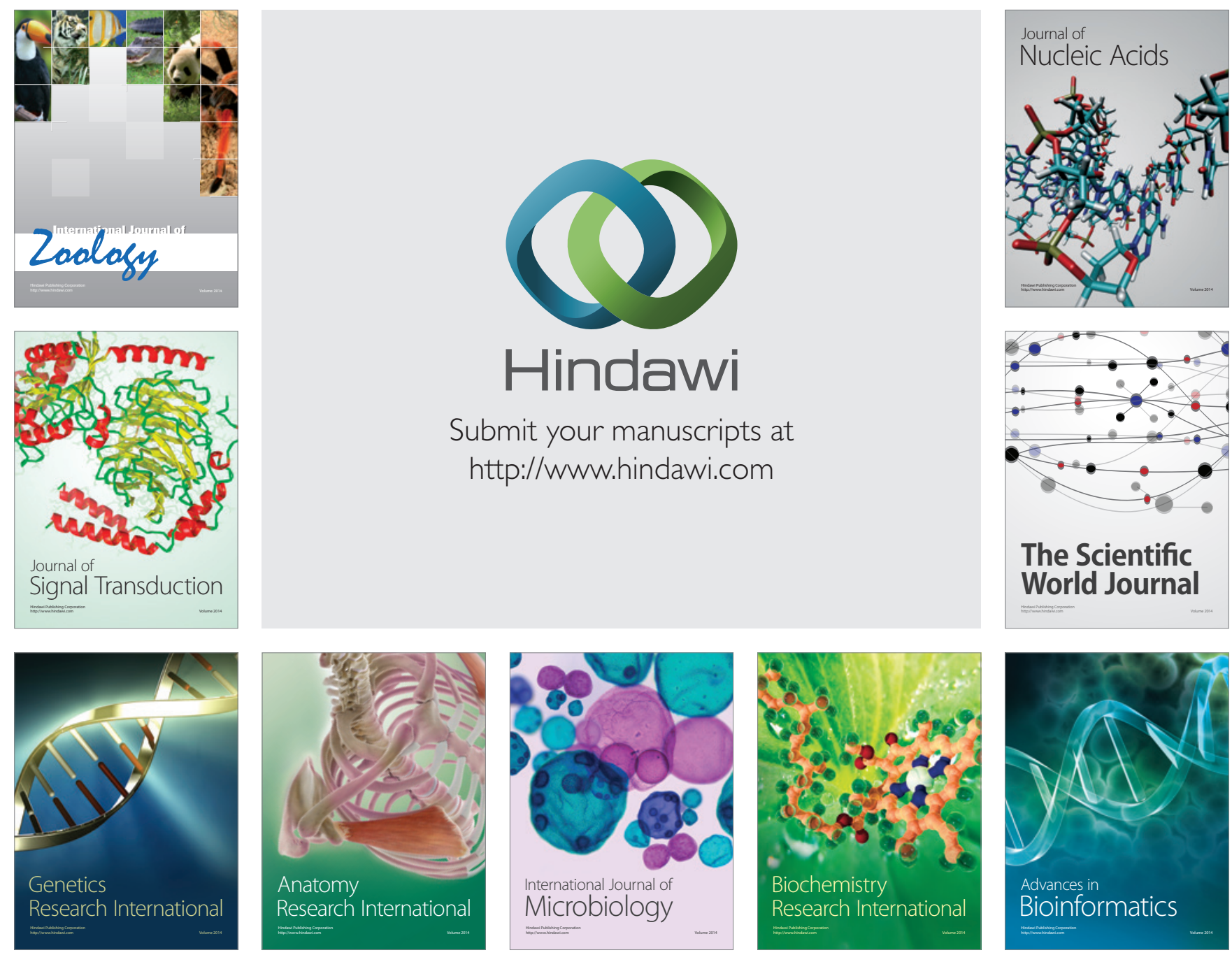

The Scientific World Journal
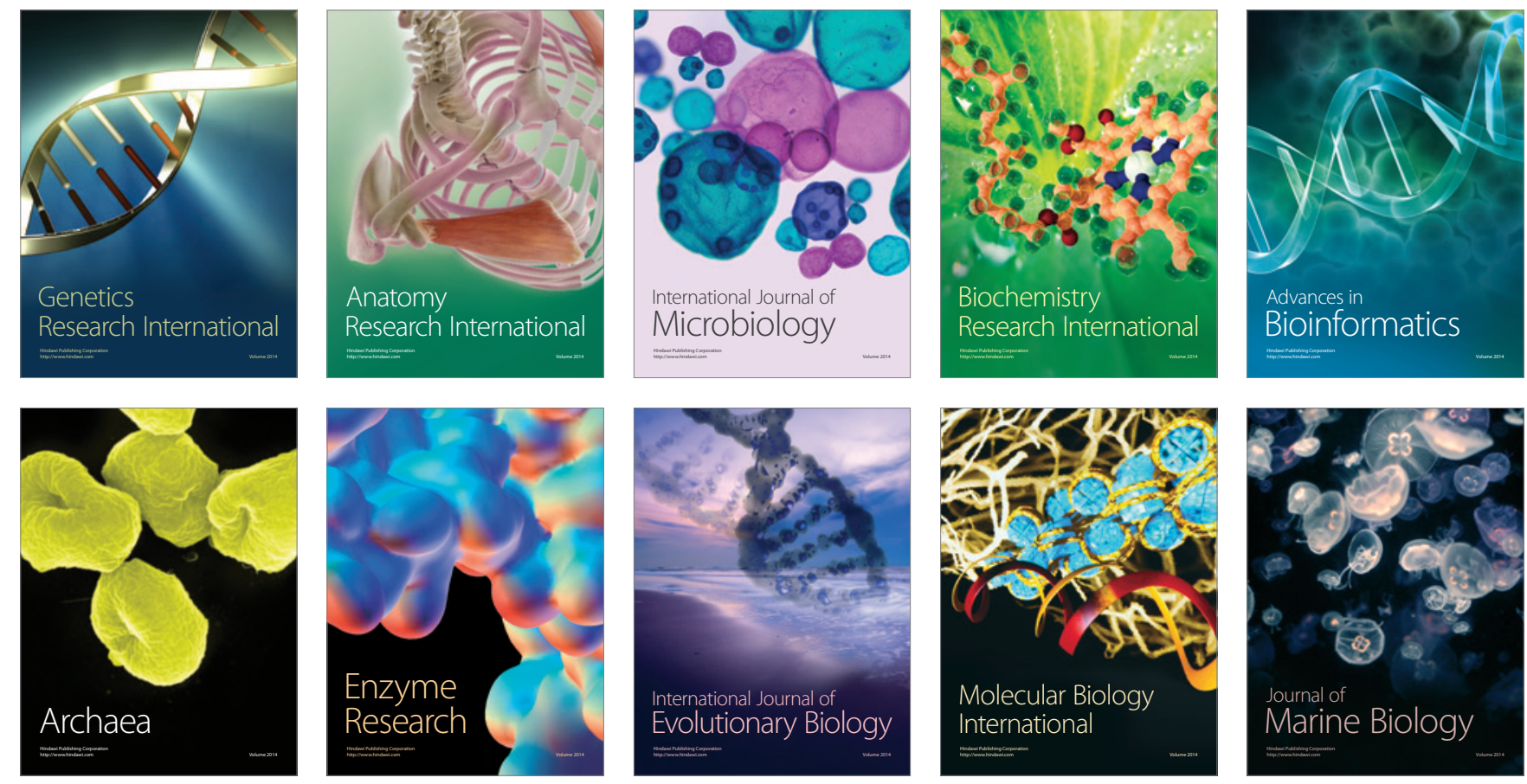